\title{
Measuring the Size of the Vela Pulsar's Radio Emission Region
}

\author{
C.R. Gwinn
}

Physics Department, UC Santa Barbara, Santa Barbara, California, USA

J.E. Reynolds, D.L. Jauncey

Australia Telescope National Facility, Epping, New South Wales, Australia

H. Hirabayashi, H. Kobayashi, Y. Murata, P.G. Edwards

Institute of Space and Astronautical Science, Sagamihara, Kanagawa, Japan

B. Carlson, S. Dougherty, D. Del Rizzo

Dominion Radio Astronomy Observatory, Hertzberg Institute of Astrophysics, National Research Council of Canada, Penticton, British Columbia, Canada

\section{M.C. Britton}

Swinburne Centre for Astrophysics and Supercomputing, Swinburne University of Technology, Hawthorn, Victoria, Australia

P.M. McCulloch, J.E.J. Lovell

Physics Department, University of Tasmania, Hobart, Tasmania, Australia

\begin{abstract}
We describe the expected distribution of intensity for a scintillating source of finite size observed through a scattering medium, including systematic and instrumental effects. We describe measurements of the size of the Vela pulsar, using this technique.
\end{abstract}

\section{Theoretical Background}

Waves from a pointlike source observed through a scattering medium will suffer random phase changes. If the phase changes are much larger than 1 radian, the observer will receive radiation from many Fresnel zones, and the scattering is said to be "strong". In this case the electric field at the plane of the observer is the sum of the electric field from many lines of sight, differing random phases (Goodman 1985). The net electric field is the result of a random walk. The electric field is thus drawn from a Gaussian distribution. Its square modulus, the intensity, is drawn from an exponential distribution (Scheuer 1968). 

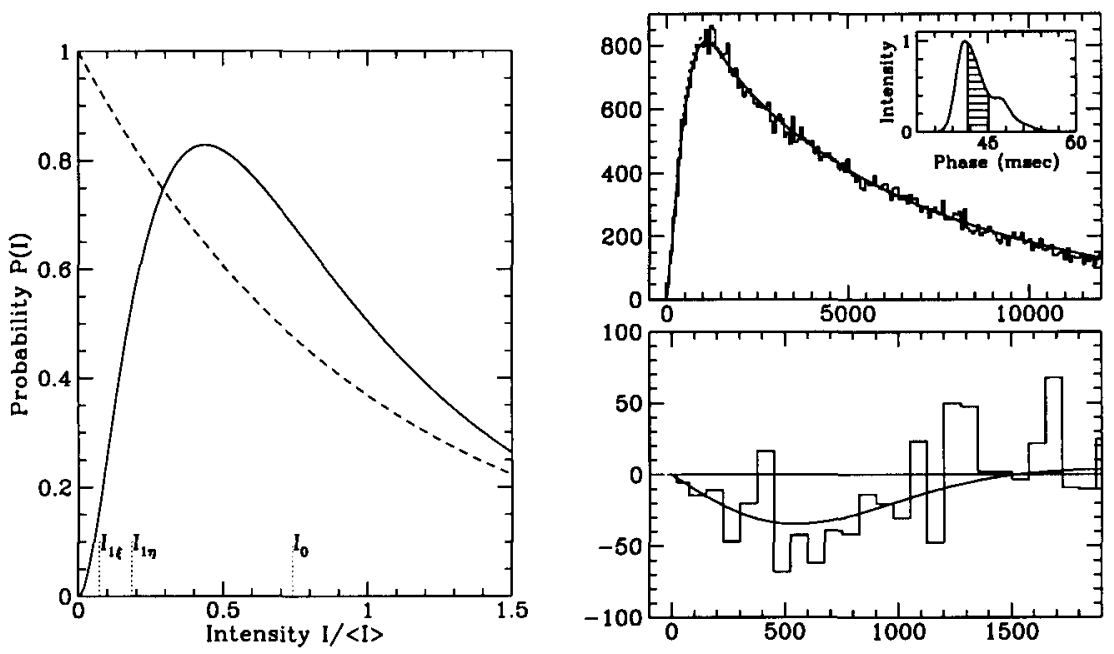

Figure 1. Left: Expected distribution of intensity for a point source in strong scintillation (dashed line); and for a source of small but finite size (solid line). From Gwinn et al. (1998). Right upper: Observed distribution of correlated flux density on a short baseline, for the Vela pulsar. Lower: Histogram shows residual to the best-fitting distribution for point source, taking into account the expected noise level. Solid curve shows best-fitting model including source size. From Gwinn et al. (2000a).

The region from which the observer receives radiation is known as the scattering disk. Scattering changes phases in the Fresnel zones, and thus acts somewhat like a lens. If the source is resolved by this "lens", the observed intensity is an incoherent sum from each part of the source. For a source of small but finite size, the resulting distribution of intensity is the sum of 3 exponentials. The scales of the smaller exponentials are approximately the size of the source along either direction on the sky, in units of the linear resolution of the scattering disk (Gwinn et al. 1998). Figure 1 shows example of the resulting intensity distributions for a point source, and for a small but resolved source. When the source is resolved, the lowest intensities are absent.

\section{Observations}

We compare the observed distribution of intensity with theoretical models to find the size of the Vela pulsar. The Vela pulsar is a favorable object for such observations because it is strong and heavily scattered. Observations at decimeter wavelengths easily capture many independent scintles in time and frequency. We observe the source interferometrically, rather than with a single dish, to avoid interference and effects of the substantial noise baseline seen in single-dish observations. Details of the observations are described elsehwere (Gwinn et al. 2000a). 
Figure 1 shows an example of the observed distribution of correlated flux density on the short Tidbinbilla-Parkes baseline for the Vela pulsar. We find a size of $340 \pm 80 \mathrm{~km}$ for the data shown in the figure.

Noise affects the distribution shown in Figure 1 strongly. Like finite source size, noise reduces the number of points at small amplitude. Noise can be measured accurately from observations of quasars, blank sky, or between pulses. Its effects can then be removed. The effects of changes in spectral structure on noise from digitization can also be caculated (Gwinn et al. 2000b).

Several effects other than noise can also affect the observed distribution. Among these are correlator saturation, shot noise, pulse-to-pulse variability, and gain variations. These can be either calculated theoretically, measured from observations, or inferred from the distribution of intensity. Gwinn et al. (2000a) discuss these effects in detail.

\section{Modulation Index}

The fact that source size affects the distribution of intensity, in scintillation, has long been known. ("Stars twinkle, planets do not.") The modulation index, $m=\sqrt{\left\langle I^{2}\right\rangle-\langle I\rangle^{2}} /\langle I\rangle$, quantifies the effect (Salpeter 1967, Cohen, Gundermann, \& Harris 1967). For a point source $m=1$; for an extended source $m<1$, with smaller modulation $m$ for a larger source, other factors being equal. Single-dish observers used measurements of modulation index to infer source sizes before the advent of radio interferometry, and this technique remains standard at low frequencies (Hewish, Readhead, \& Duffett-Smith 1974, Hajivasiliou 1992). However, it is more subject to scintillation shot noise, and less immune to systematic effects, than a direct comparison of distribution functions.

A finite observation necessarily samples a finite number of scintles. Averages over this sample approximate the statistical averages $\left\langle I^{2}\right\rangle$ and $\langle I\rangle$. Because the nearly-exponential distribution falls off rapidly at high intensity, these sums (particularly $<I^{2}>$ ) are dominated by the relatively rare scintles with the highest intensities. On the other hand, the effects of source structure are most important at the lowest intensities, where the number of scintillations is large, but the contribution to $\langle I\rangle$ and $\left\langle I^{2}\right\rangle$ is small. Thus, direct estimation of the modulation index is relatively insensitive to source size and relatively more sensitive to scintle shot noise than a direct comparison of the forms of distribution functions.

Correlator saturation also affects the modulation index strongly, because its effects are largest at high intensity. Moreover, since the observable is a single number, rather than a distribution, it is more difficult to know what effects are playing signficant roles.

Interestingly, Roberts \& Ables (19) measured the modulation index, as well as the characteristic time and frequency scales of scintillation, in their classic study of scattering of southern-hemisphere pulsars. They report a modulation index of $0.97 \pm 0.03$ for the Vela pulsar at $18 \mathrm{~cm}$ wavelength, and of $0.90 \pm 0.02$ at $9 \mathrm{~cm}$ wavelength. Interpolation between these values is consistent with our results quoted above.

Interestingly, Roberts \& Ables find that the modulation index is smaller at shorter observing wavelengths, suggesting that the source size is greater. This 
conclusion is surprising from the standpoint of the standard radius-to-frequency mapping. (Note, however, that these measurements are of size rather than emission height.) The larger inferred size might reflect on the more complicated pulse profile of this pulsar at shorter wavelengths (Kern \& Hankins 2000). On the other hand, it might also reflect systematic effects; at short wavelengths the scintles have wide bandwidths but the source remains quite strong, so that correlator saturation should become more serious. In contrast, self-noise and gain variations might be expected to be more important at lower frequencies. Observations of the full distribution of intensity in scintillation, as a function of wavelength, should indicate the origin of this variation of modulation index.

\section{References}

Cohen, M.H., Gundermann, E.J., \& Harris, D.E. 1967, ApJ, 150, 767

Goodman, J.W. 1985, Statistical Optics, New York: Wiley

Gwinn, C.R., Britton, M.C., Reynolds, J.E., Jauncey, D.L., King, E.A., McCulloch, P.M., Lovell, J.E.J., \& Preston, R.A. 1998, ApJ, 505, 928

Gwinn, C.R., Britton, M.C., Reynolds, J.E., Jauncey, D.L., King, E.A., McCulloch, P.M., Lovell, J.E.J., Flanagan, C.S., \& Preston, R.A. 2000, ApJ, in press

Gwinn, C.R., Britton, M.C., Carlson, B., Dougherty, S., Del Rizzo, D., Reynolds, J.E., Jauncey, D.L., McCulloch, P.M., Hirabayashi, H., Kobayashi, H., Murata, Y., \& Edwards, P.G. 2000, in preparation

Hajivassiliou, C.A. 1992, Nature, 355, 232

Hewish, A., Readhead, A.C.S., \& Duffett-Smith, P.J., 1974, Nature, 252, 657

Kern, J., \& Hankins, T. 2000, these proceedings

Roberts, J.A., \& Ables, J.G. 1982, MNRAS, 201, 1119

Salpeter, E.E. 1967, ApJ, 147, 433

Scheuer, P.A.G. 1968, Nature, 218, 920 